\title{
Therapeutic Management of Myiasis Wound due to Traumatic Injury in Gingival of a Murrah Buffalo Calf
}

\author{
Praveen Kumar ${ }^{1 *}$, Anup Yadav ${ }^{1}$, Lokesh $^{1}$, Umed Singh Mehra ${ }^{1}$, \\ Rajendra Yadav ${ }^{2}$ and Pankaj Kumar ${ }^{3}$
}

\author{
${ }^{1}$ Department of Animal Husbandry and Dairying, Govt. of Haryana, India \\ ${ }^{2}$ Regional Veterinary Diagnostic and Extension Centre, Mahendergarh (LUVAS, Hisar), \\ Haryana, India \\ ${ }^{3}$ Disease Investigation Laboratory, Rohtak (LUVAS, Hisar), Haryana, India
}

*Corresponding author

\begin{abstract}
A B S T R A C T
Keywords

Therapeutic management, Myiasis wound, Gingival, Murrah buffalo calf

Article Info

Accepted:

22 October 2018

Available Online:

10 November 2018

\section{Introduction}

Myiasis or maggot wound refers to the parasitic infestation of tissue of a living vertebrate animal by larvae of the order Diptera (true flies) that grow inside the host while feeding on its dead or living tissue causing more or less every traumatizing injury depending on the relationship of the larvae with the host and the location (Hall and Farkas, 2000; Francesconi and Lupi, 2005; Robbins and Khachemoune, 2010). Clinically, myiasis can be classified based on the area of the body affected by the maggot or subsequent
\end{abstract}

Myiasis in all species of animals is very common during the rainy season. The present case report emphasizes on such one interesting case in a 20 day old male buffalo calf infested with maggots in gingival region. The calf was successfully treated with rinsing by normal saline solution, turpentine oil, antiseptic solution and ivermectin injection. development in the host (Francesconi and Lupi, 2006), which may include gastrointestinal, urogenital, ocular, nasopharyngeal, auricular and cutaneous myiasis (Abdullah et al., 2015). Therapeutic management of the myiasis wounds includes mechanical removal of maggots or by application of wound cleansing agents and/or a combination of both followed by general wound management. Removal of necrotic tissue abolishes many of the associated bacteria too (Rafee et al., 2016). Current methods used to treat wounds include debridement, irrigation, antibiotics, tissue 
grafts, proteolytic enzymes and corticosteroids, sometimes, which also possess major drawbacks and unwanted side effects (Nayak et al., 2010). Though, the proper method for the treatment of maggot wound is not well defined (Caissie, 2008), but depending upon the species and site of infestation a veterinarian may handle the case in best possible way by removal of maggots, local dressing of the affected tissue and use of parenteral therapy. Although cutaneous myiasis is the most commonly reported form of the maggot wound in the tropics (Abdullah et al., 2015), myiasis of the gingival region in buffalo calf is rarely reported. Knowledge of the disease is imperative from the preventive, diagnostic and curative point of view even though the disease is not a lethal disorder. This case reports a case myiasis wound due to traumatic injury in gingival of a Murrah buffalo calf.

\section{Case history and clinical observations}

A 20 day old, intact male Murrah buffalo calf was presented at Government Veterinary Hospital Hudina (Mahendergarh, Haryana) with the history of persistent drop wise bleeding from the mouth since 3 days along with difficulty in suckling of milk. There was a history of traumatic injury in the gingival region of the calf by a blunt object. Thorough clinical examination of the animal revealed presence of maggots at the gingival base in lower jaw with profuse bleeding and tarring of gingival (Fig. 1). Vital body parameters of the calf such as rectal temperature, respiration rate and pulse rate were within the normal limits. Clinical findings confirm that buffalo calf was infested with myiasis wound in gingival region.

\section{Therapeutic management and recovery}

The maggot wound was rinsed with normal saline water to wash away the dead maggots present on the surface of wound. Rinsing was done under pressure using disposable syringe and repeated 3-4 times. The remaining maggots were gently wiped away or removed with a pair of tweezers or artery forceps. The owner was advised to keep the wound moist by placing clean bandage soaked normal saline solution repeatedly over the wound as many as times possible. The owner was also advised to apply an antiseptic and fly repellent solution on the wound daily twice a day. After 2 days a gauze bandage soaked with turpentine oil was kept on the open wound for about 10 minutes. This helped to draw out and kill maggots that have burrowed deeply into the wound. The wound was again rinsed with normal saline water to wash away the maggots that have worked to the surface of the wound. The remaining maggots were again gently wiped away and removed with a pair of tweezers or artery forceps. On the same day, Ivermectin (Inj. Hitek) @ $0.3 \mathrm{mg} / \mathrm{kg}$ body weightwas administered once by subcutaneous route. The supportive therapy for this case was includes administration of Flunixinmeglumine (Inj. Megludyne) @ $1.1 \mathrm{mg} / \mathrm{kg}$ body weigh intramuscularly once a day for 3 days as antiinflammatory, anti-pyrexic and analgesic agent. Long acting antibiotic oxytetracycline (Inj. Oxytetracycline-LA) @ 20mg/kg) was also given once deep intramuscularly to treat secondary bacterial infections. After 5 days of treatment, the buffalo calf recovered fully indicated by absence of maggots and bleeding in gingival region, proper suckling of milk and normal physical activities (Fig. 2). Almost similar line of treatment was adopted by Abdullah et al., (2015) during the clinical management of cutaneous myiasis wound due to post traumatic horn injury in a bull. Although myiasis is common in tropical countries like India, but the location of myiasis wound due to traumatic injury in gingival region of a buffalo calf as reported in this case is rare as there are no available references in the literature. 
Int.J.Curr.Microbiol.App.Sci (2018) 7(11): 2979-2983

Fig.1 Buffalo calf infested with myiasis in gingival region

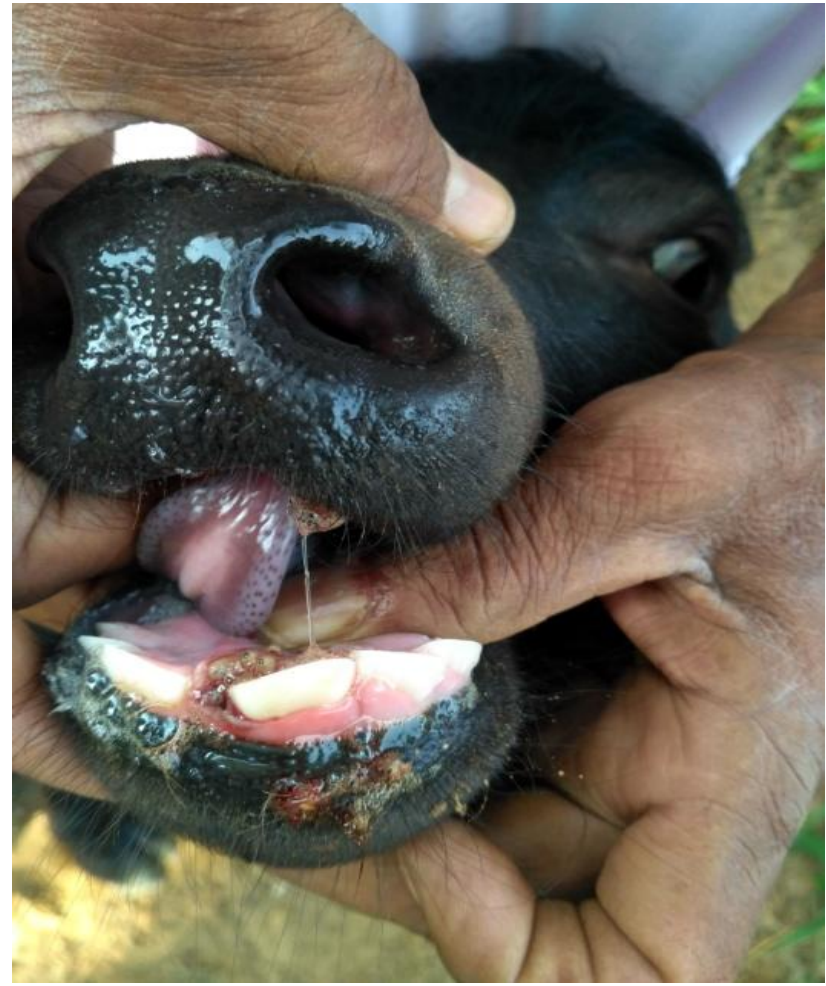

Fig.2 Buffalo calf after the treatment

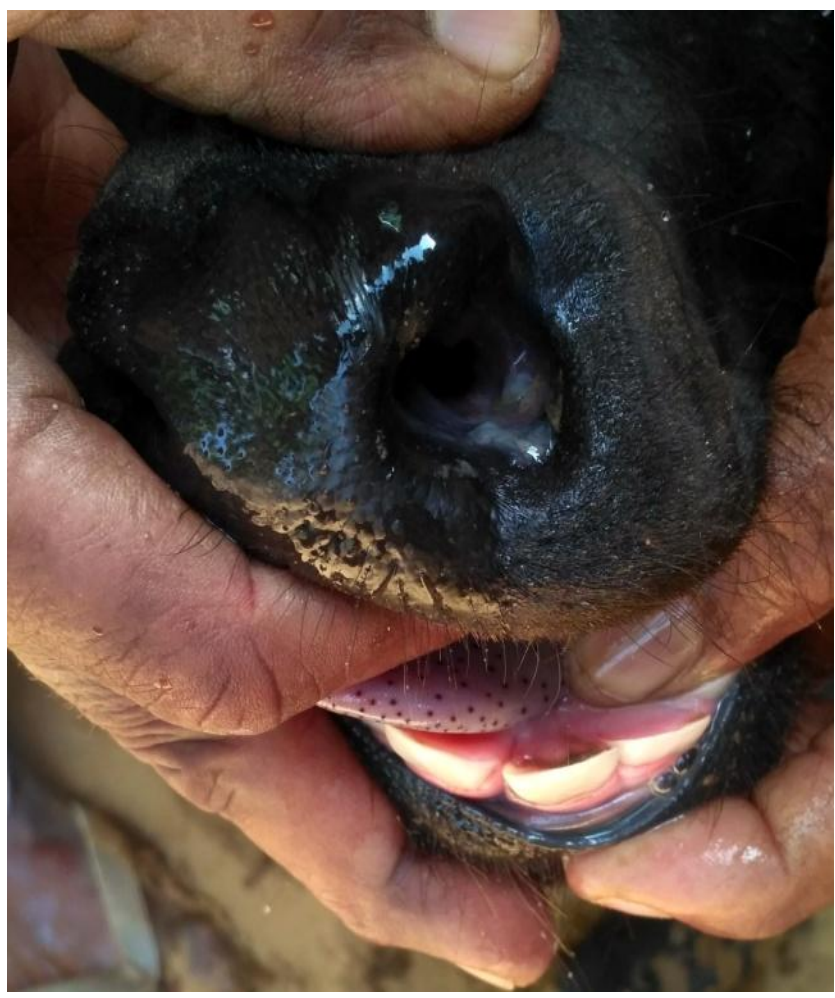


Though the myiasis is not a lethal problem, Knowledge about the disease is of vital importance from the preventive, diagnostic and curative point of view. For animal with traumatic wound as reported in this case, proper treatment of the wound needs to be done promptly to prevent further complications. Hygiene of the farm needs to be considered where proper management of fecal materials can be practised to prevent overproduction of flies (Bowler et al., 2001). Clinical presentation of myiasis may vary depending on the specific cause and the body part affected like skin, ocular, nasal, oral, gastrointestinal, aural and genitourinary tracts (Kpea and Zywocinski, 1995).

In the present case, the calf was presented with traumatic injury in the gingival region of lower jaw where maggots were seen on the wound. Application of a toxic substance to the larva and egg, production of localized hypoxia to force the emergence of the larva, mechanical or surgical removal of the maggots from the site of infestation and surgical debridement are the various therapeutic measures indicated for the management of various types of myiasis (Francesconi and Lupi, 2006).

After removal of the larvae and dead tissues, the wound was dressed daily with antiseptic solution, administration of ivermectin and systemic administration of antibiotic against secondary bacterial infection were done in this case and in agreement with Falish (2012) and Abdullah et al., (2015).

The present case report concluded that myiasis in domestic animals can affect sometimes the unusual body parts like gingival region also. The case presented with maggot wound in a Murrah buffalo calf was effectively clinically managed by standard way of the treatment of myiasis and there was uneventful recovery.

\section{References}

Abdullah, F.F.J., Chung, E.L.T., Marza, A.D., Mohammed, K., Abba, Y., Tijjani, A. and Sadiq, M.A. (2015). Clinical management of cutaneous myiasis wound due to post traumatic horn injury in a bull: A case report. J. Agri. Vet. Sci. 8(11): 73-75.

Bowler, P., Duerden, B. and Armstrong, D. (2001). Wound microbiology and associated approaches to wound management. Clin.Microbio.Rev. 14: 244-269.

Caissie, R. (2008). Cutaneous myiasis: diagnosis, treatment and prevention. J.Oral. Maxillofac. Surg. 66: 560-568.

Falish, G. (2012). Wound management for veterinary technicians. In: Illinois state veterinary medical association (ISVMA) annual conference proceedings 2012. Illinois state veterinary medical association (ISVMA), Westin Lombard Yorktown Center, 70 Yorktown Center, Lombard, IL 60148, Illinois, USA.

Francesconi, F. and Lupi, O. (2005). Myiasis. Clin. Microbio. Rev. 25(1): 79-105.

Francesconi, F. and Lupi, O. (2006). Tropical Dermatology, Elsevier, Philadelphia.

Hall, M.J. and Farkas, R. (2000). Traumatic myiasis of humans and animals. In: Contributions to a Manual of Palaearctic Diptera. Science Herald, Budapest. pp: 751-768.

Kpea, N. and Zywocinski, C. (1995). Flies in the flesh: a case report and review of cutaneous myiasis. Cutis. 55(1): 47-48.

Nayak, B.S., Julien, R.M. and Godwin, I. (2010). Wound healing potential of ethanolic extract of Kalanchoepinnata Lam. leaf- A preliminary study. Indian J. experiment. biology. 48: 572-576.

Rafee, M.A., Amarpal, Kinjavdekar, P. and Aithal, H.P. (2016). A protocol for the successful management of maggot 
wound in dogs.Indian J. Canine Pract. 6: $141-143$. common types of myiasis. Int. J. Dermatology. 49(10): 1092-1098.

Robbins, K. and Khachemoune, A. (2010).

Cutaneous myiasis: a review of the

\section{How to cite this article:}

Praveen Kumar, Anup Yadav, Lokesh, Umed Singh Mehra, Rajendra Yadav and Pankaj Kumar. 2018. Therapeutic Management of Myiasis Wound due to Traumatic Injury in Gingival of a Murrah Buffalo Calf. Int.J.Curr.Microbiol.App.Sci. 7(11): 2979-2983. doi: https://doi.org/10.20546/ijcmas.2018.711.341 